\title{
Crystallizable and Tough Aliphatic Thermoplastic Polyureas Synthesized through a Non-isocyanate Route
}

Suqing Li, Zhihui Sang, Jingbo Zhao*, Zhiyuan Zhang, Junying Zhang, and Wantai Yang

Key Laboratory of Carbon Fiber and Functional Polymers(Beijing University of Chemical Technology), Ministry of Education; State Key Laboratory of Chemical

Resource Engineering; College of Materials Science and Engineering, Beijing University of Chemical Technology, Beijing 100029, China

Corresponding author: Jingbo Zhao (zhaojb@mail.buct.edu.cn), Tel/Fax: +8610-6443-4864 


\section{Purification and Analysis of the Impurities in TPUrea-1}

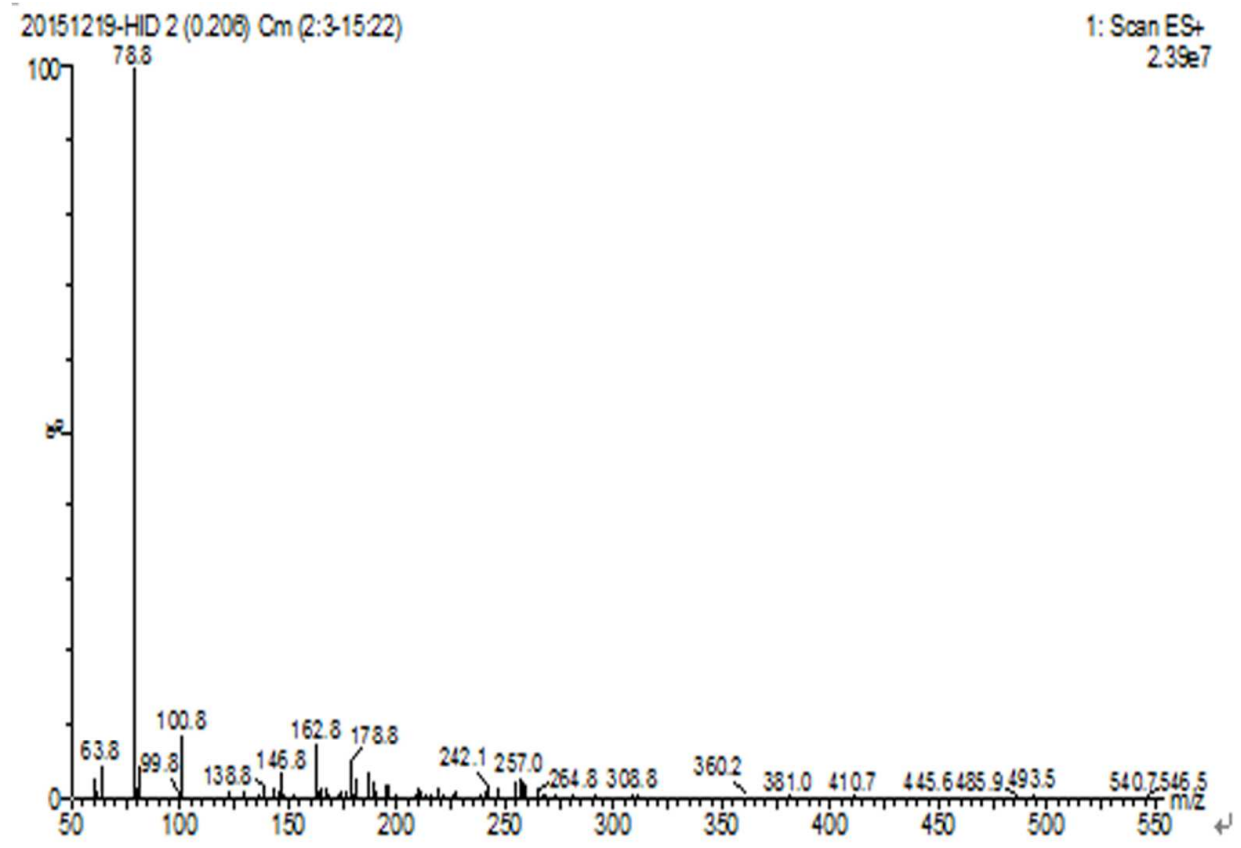

Figure S1. ESI-MS spectrum of the concentrated filtrate in TPUrea-1 purification

To analyze the impurities which influence the determination of TPUrea-1 with NMR or FT-IR spectra, TPUrea-1 was purified thrice through dissolution-precipitation cycles by using $20 \mathrm{~mL}$ DMSO as solvent and $200 \mathrm{~mL}$ ether as non-solvent. The filtrate was collected and evaporated under reduced pressure. The concentrated filtrate was analyzed by ESI-MS. Figure S1 shows the ESI-MS spectrum of the concentrated filtrate in TPUrea-1 purification. The major component of the concentrated filtrate is DMSO $(\mathrm{M}+1,79 ; \mathrm{M}+23,101)$, which was used as solvent in TPUrea-1 purification. Some impurities were also included in the concentrated filtrate, i.e. glycol $(\mathrm{M}+1,63)$ which was formed in the synthesis reaction of TPUrea-1, 1,6-hexanediamine (HAD) $(M+23,139)$ which might be formed from the decomposition of BHHDU, and some unknown compounds. The impurities are in low level. As NMR and FTIR analysis is highly sensitive to impurities, purification is necessary before NMR detection. 


\section{Cooling and Second Heating DSC Data of TPUreas at Different Cooling Rates}

Table S1. Cooling and second heating DSC data of TPUreas at different cooling rates (heating rate: $10^{\circ} \mathrm{C} \cdot \mathrm{min}^{-1}$ under $\mathrm{N}_{2}$ )

\begin{tabular}{|c|c|c|c|c|c|c|c|}
\hline \multirow{3}{*}{ Sample } & \multicolumn{3}{|c|}{ Cooling period } & \multicolumn{4}{|c|}{ Second heating } \\
\hline & Cooling rate/ & $T_{\mathrm{cc}}{ }^{\mathrm{a}} \rho \mathrm{C}$ & $\Delta H_{\mathrm{cc}}^{\mathrm{a}} /$ & $T_{\mathrm{c}}^{\mathrm{b}} /$ & $\Delta H_{\mathrm{c}}^{\mathrm{b}} /$ & $T_{\mathrm{m}} /$ & $\Delta H_{\mathrm{m}}$ \\
\hline & ${ }^{\circ} \mathrm{C} \cdot \min ^{-1}$ & & $\mathrm{~J} \cdot \mathrm{g}^{-1}$ & ${ }^{\circ} \mathrm{C}$ & $J \cdot g^{-1}$ & ${ }^{\circ} \mathrm{C}$ & $J \cdot g^{-1}$ \\
\hline \multirow[t]{4}{*}{ TPUrea-1 } & 10 & -- & -- & -- & -- & -- & -- \\
\hline & 20 & -- & -- & -- & -- & -- & -- \\
\hline & 30 & -- & -- & -- & -- & -- & -- \\
\hline & 40 & -- & -- & -- & -- & -- & -- \\
\hline \multirow[t]{4}{*}{ TPUrea-2 } & 10 & -- & -- & 58.8 & 12.7 & 142.8 & 16.5 \\
\hline & 20 & -- & -- & 59.3 & 13.5 & 139.7 & 15.2 \\
\hline & 30 & -- & -- & 59.7 & 13.9 & 140.5 & 16.4 \\
\hline & 40 & -- & -- & 59.5 & 13.7 & 140.5 & 17.6 \\
\hline \multirow[t]{4}{*}{ TPUrea-3 } & 10 & 61.5 & 12.8 & -- & -- & 142.8 & 14.3 \\
\hline & 20 & -- & -- & 50.2 & 12.1 & 141.2 & 16.2 \\
\hline & 30 & -- & -- & 49.7 & 13.6 & 141.3 & 19.1 \\
\hline & 40 & -- & -- & 50.6 & 14.7 & 141.5 & 17.4 \\
\hline \multirow[t]{4}{*}{ TPUrea-4 } & 10 & 115.1 & 7.8 & -- & -- & 157.8 & 12.6 \\
\hline & 20 & 103.8 & 8.9 & -- & -- & 156.8 & 13.8 \\
\hline & 30 & 82.3 & 13.9 & -- & -- & 150.8 & 17.2 \\
\hline & 40 & 77.5 & 14.8 & -- & - & 149.5 & 19.2 \\
\hline \multirow[t]{4}{*}{ TPUrea-5 } & 10 & 118.2 & 16.5 & -- & -- & 159.4 & 10.3 \\
\hline & 20 & 104.2 & 17.0 & -- & -- & 154.9 & 12.9 \\
\hline & 30 & 102.4 & 18.5 & -- & -- & 155.5 & 13.3 \\
\hline & 40 & 87.0 & 19.6 & -- & -- & 149.8 & 17.1 \\
\hline
\end{tabular}

${ }^{\mathrm{a}} \mathrm{T}_{\mathrm{cc}}$ and $\Delta H_{\mathrm{cc}}$ : cooling crystallization temperature and the enthalpy determined in cooling period.

${ }^{\mathrm{b}} T_{\mathrm{c}}$ and $\Delta H_{\mathrm{c}}$ : crystallization temperature and the enthalpy determined in the second heating DSC scan. 